Bundesgesundheitsbl 2016 $59: 543-544$

DOI 10.1007/s00103-016-2344-5

Online publiziert: 18. April 2016

๑) Springer-Verlag Berlin Heidelberg 2016

CrossMark

\section{Oliver Razum ${ }^{1}$ - Anke-Christine Saß $\aleph^{2}$ Kayvan Bozorgmehr ${ }^{3}$}

${ }^{1}$ AG Epidemiologie und International Public Health, Fakultät für Gesundheitswissenschaften, Universität Bielefeld, Bielefeld, Deutschland

${ }^{2}$ Abteilung für Epidemiologie und Gesundheitsmonitoring, Robert Koch-Institut, Berlin, Deutschland

${ }^{3}$ Abteilung Allgemeinmedizin und Versorgungsforschung, Universitätsklinikum Heidelberg, Heidelberg, Deutschland

\title{
Gesundheitliche Versorgung von Geflüchteten: Herausforderungen und Lösungsansätze
}

Über eine Millionen Menschen haben im Jahr 2015 in Deutschland Schutz gesucht - vor Krieg und Verfolgung oder vor einer als hoffnungslos empfundenen wirtschaftlichen Situation in ihrem Herkunftsland. Um nach Deutschland zu gelangen, haben sie oft lange und ihre Gesundheit beeinträchtigende Wege auf sich genommen. Eine große Zahl von Menschen hat auf der Flucht ihr Leben verloren: Laut dem „Missing Migrant Project“ der International Organisation for Migration (IOM) gab es 2015 allein im Mittelmeerraum 3770 Todesfälle. Es steht $\mathrm{zu}$ vermuten, dass die Fluchtmigration unter gesundheitsgefährdenden Bedingungen weiter anhalten und Deutschland auch in den kommenden Jahren Ziel vieler Geflüchteter sein wird.

Die angemessene Versorgung dieser Menschen stellt die Gesundheitsdienste vor große und in dieser Form noch nicht dagewesene Herausforderungen. Mit dem vorliegenden Schwerpunktheft möchten wir versuchen, die vorhandene Evidenz oder zumindest die besten verfügbaren Erfahrungen („Best Practice“) aus den Bereichen medizinische Versorgung, Prävention und der Stärkung lokaler Gesundheitssystemstrukturen aufzuzeigen.

\section{Angemessene Strategien entwickeln, bewährte übernehmen}

Das Vorgehen bei der Erstuntersuchung geflüchteter und asylsuchender Menschen, bei deren Behandlung und bei der Umsetzung von präventiven Maßnahmen wie Impfungen ist in Deutschland nicht standardisiert. Öffentlicher Gesundheitsdienst (ÖGD) und Kommunen entwickeln auf Basis der ihnen zur Verfügung stehenden Ressourcen die bestmöglichen Lösungsstrategien. Das zeigen Bozorgmehr et al. in einer deutschlandweiten Befragung von Gesundheitsämtern. Erforderlich wären ein stärker koordiniertes und standardisiertes Vorgehen bei Impfungen, bei der Erhebung und datenschutzkonformen Übermittlung gesundheitsbezogener Informationen sowie eine Konzentration auf wenige, bevölkerungsmedizinisch wichtige Infektionskrankheiten bei der Erstuntersuchung. Gardemann und Wilp halten es für ethisch wie praktisch geboten, hierzu die bewährten normativen und technischen Standards der internationalen humanitären Not- und Soforthilfe heranzuziehen.

Geflüchtete und Asylsuchende erhalten nach $\$ \$ 4$ und 6 des Asylbewerberleistungsgesetzes zunächst nur eingeschränkten Zugang zur Gesundheitsversorgung. Erst nach 15-monatigem Aufenthalt in Deutschland stehen ihnen die regulären Leistungen der gesetzlichen Krankenversicherung vollumfänglich zur Verfügung. Derzeit erhalten Asylsuchende nur in Bremen und Hamburg ohne Wartezeit eine Gesundheitskarte und damit einfacheren sowie diskriminierungsfreien Zugang zur Versorgung. Mohammadzadeh et al. stellen das Bremer Modell vor, das eine frühe umfassende Versorgung mit primärmedizinischen Maßnahmen sicherstellt. Es ist ein Modell, in welchem der ÖGD durch ein niedrigschwelliges Versorgungsangebot als "gate opener" zur Regelversorgung fungiert und somit eine zentrale statt einer subsidiären Rolle in der Versorgung geflüchteter Menschen spielt.

\section{Infektionskrankheiten: Risiken realistisch einschätzen und Prävention effektiv umsetzen}

In der Bevölkerung besteht Sorge, dass Geflüchtete Infektionskrankheiten nach Deutschland bringen, die sich dann hier ausbreiten könnten. Tatsächlich aber sind Geflüchtete eher durch den Aufenthalt in Massenunterkünften oder durch den Kontakt mit ungeimpften Deutschen gefährdet, wenn sie aufgrund kriegerischer Auseinandersetzungen beispielsweise keinen Impfschutz gegen Windpocken oder Masern erhalten konnten. Sie sind also eher „at risk“ als „of risk“, wie Kühne und Gilsdorf anhand von Untersuchungen von Ausbrüchen in Gemeinschaftsunterkünften zeigen. 
Zwar ist die Prävalenz einzelner Infektionskrankheiten bei Einreise höher als in der deutschen Mehrheitsbevölkerung, wie Hampel et al. anhand der Hepatitis bei Geflüchteten darlegen. Die eigentliche Herausforderung bei dieser und vergleichbaren Erkrankungen, so die Autorinnen und Autoren, liegt jedoch in der kontinuierlichen Versorgung und Anbindung der Geflüchteten an das deutsche Gesundheitssystem. Wie Zeitlmann et al. am Beispiel der Polioimpfung von syrischen Flüchtlingen während des Polioausbruchs 2013 in Syrien zeigen, unternehmen Erstaufnahmeeinrichtungen große Anstrengungen bei der Impfprävention. Jedoch konnten nur 8 von 20 Erstaufnahmeeinrichtungen bei syrischen Kleinkindern eine Impfabdeckung von $\geq 80 \%$ erzielen. Daher ist das "Best-Practice“-Konzept, das Brockmann et al. zur Umsetzung der amtlich empfohlenen Schutzimpfungen bei Asylsuchenden vorstellen, so wichtig. Es beinhaltet die Koordination der beteiligten Partner und die Bereitstellung von Impfstoffen durch den ÖGD sowie die Kooperation mit der Ärzteschaft.

\section{Versorgungslücken identifi- zieren, Barrieren abbauen, Nutzung verbessern}

Da standardisierte Daten im Sinne einer Gesundheitsberichterstattung $\mathrm{zu}$ Geflüchteten und Asylsuchenden fehlen, ist über deren Bedarfe und mögliche Defizite bei ihrer gesundheitlichen Versorgung $\mathrm{zu}$ wenig bekannt. Empirische Studien können helfen, die bestehenden Wissenslücken zumindest zu verkleinern, wie Bozorgmehr et al. anhand einer systematischen Literaturdurchsicht zeigen. In den Bereichen chronische Erkrankungen, Kinder- und Müttergesundheit fehlen aber weiterhin grundlegende Informationen.

Geflüchtete sind oft traumatisiert und für sie besteht das Risiko, Traumafolgestörungen $\mathrm{zu}$ entwickeln. Einer angemessenen Therapie stehen ab oft aufenthaltsrechtliche Hindernisse entgegen, wie Böttche et al. erläutern. Ein Abbau solcher Barrieren würde helfen, der Entwicklung weiterer somatischer und psychischer Erkrankungen vorzubeu- gen. Zugangsbarrieren sind möglicherweise auch der Grund, weshalb Kinder von Eltern mit unsicherem aufenthaltsrechtlichen Status überdurchschnittlich häufig in der Notfallversorgung statt in der hausärztlichen Versorgung vorgestellt werden, wie Wenner et al. zeigen. Unbegleitete minderjährige Flüchtlinge werden dagegen eng vom Jugendamt betreut. Ihre gesundheitlichen Bedarfe liegen nach Untersuchungen von Spallek et al. vor allem im Bereich der psychischen Versorgung. Dafür sind derzeit spezialisierte psychosoziale Behandlungszentren zuständig, wie Metzner et al. erläutern. Wünschenswert wäre es, verstärkt niedergelassene Therapeuten einzubinden und eine evidenzbasierte, dolmetschergestützte Psychotherapie zu entwickeln.

\section{Politische und gesellschaftliche Veränderungen}

Um die gesundheitliche Situation der Menschen zu verbessern, die in Deutschland Schutz suchen, müssen Fluchtursachen wie Kriege und die weltweiten wirtschaftlichen und gesundheitlichen Ungleichheiten zwischen Nationen behoben werden. Hier hat die Bundesregierung 2013 (ähnlich wie andere EUStaaten) eine globale Gesundheitsstrategie verabschiedet, welche die Verantwortungsbereiche mehrerer Ministerien übergreift. Diese Strategie kann einen Beitrag dazu leisten, politische und wirtschaftliche Fluchtursachen $\mathrm{zu}$ reduzieren. Dazu muss sie entschlossen umgesetzt werden.

Was können wir tun als Ärztinnen und Ärzte sowie im Bereich von Public Health tätige Fachkräfte? Wir können uns aktiv für das Recht auf Gesundheit einsetzen. In Deutschland bedeutet das, allen Geflüchteten und Asylsuchenden möglichst schnell und unkompliziert den diskriminierungsfreien Zugang zur Regelversorgung zu ebnen, wie dies mit der Einführung der Gesundheitskarte in Bremen und Hamburg geschehen ist. Ein weiteres wichtiges Thema sind sichere Fluchtwege. Neben der Rettung aus Seenot wird hier vielfach ein Einwanderungsgesetz gefordert, das vermehrt legale Zuwanderung nach Deutschland und in die EU-Mitgliedsstaaten ermöglicht. Und wir können uns für die Einhaltung bestehender Rechte wie das Recht auf Schutz vor Krieg und Verfolgung sowie das Recht auf Asyl einsetzen - in Deutschland, aber auch in den anderen EU-Staaten.

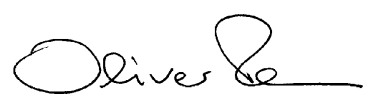

Ihr Oliver Razum

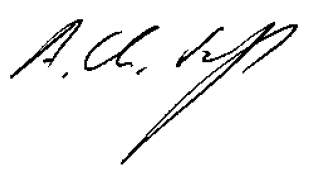

Ihre Anke-Christine Saß

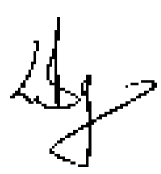

Ihr Kayvan Bozorgmehr

\section{Korrespondenzadresse}

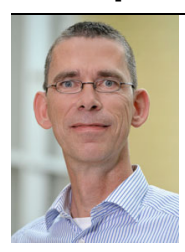

Prof. Dr. med. O. Razum

AG Epidemiologie und International Public Health, Fakultät für Gesundheitswissenschaften, Universität Bielefeld Universitätsstraße 25, 33615 Bielefeld, Deutschland oliver.razum@uni-bielefeld.de

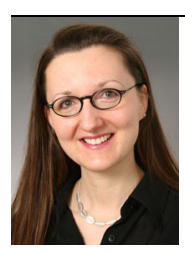

Dr. A.-C. Saß

Abteilung für Epidemiologie und Gesundheitsmonitoring, Robert Koch-Institut General-Pape-Str. 62-66, 12101 Berlin, Deutschland SassA@rki.de

Dr. med. K. Bozorgmehr
Abteilung Allgemeinmedizin
und Versorgungsforschung,
Universitätsklinikum
Heidelberg
Marsilius-Arkaden, Im
Neuenheimer Feld 130.3,
Turm West,69120 Heidelberg,
Deutschland
kayvan.bozorgmehr@med.uni-
heidelberg.de

Interessenkonflikt. O. Razum, A.-C. Saß und K. Bozorgmehr geben an, dass kein Interessenkonflikt besteht. 